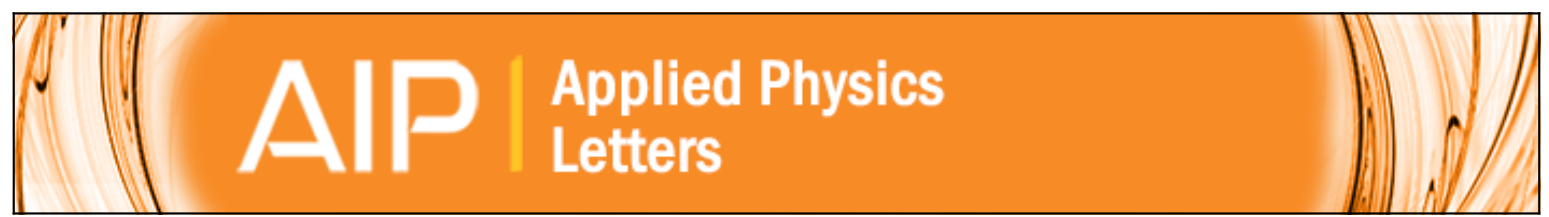

\title{
Resolving interparticle position and optical forces along the axial direction using optical coherence gating
}

T. H. Chow, W. M. Lee, K. M. Tan, B. K. Ng, and C. J. R. Sheppard

Citation: Applied Physics Letters 97, 231113 (2010); doi: 10.1063/1.3519976

View online: http://dx.doi.org/10.1063/1.3519976

View Table of Contents: http://scitation.aip.org/content/aip/journal/apl/97/23?ver=pdfcov

Published by the AIP Publishing

\section{Articles you may be interested in}

Optical forces from partially coherent light: Near field of statistically homogeneous sources

AIP Conf. Proc. 1475, 38 (2012); 10.1063/1.4750088

Analysis of micromachined Fabry-Pérot cavities using phase-sensitive optical low coherence interferometry: Insight on dimensional measurements of dielectric layers

AIP Advances 2, 022143 (2012); 10.1063/1.4727741

Simultaneous measurement of substrate temperature and thin-film thickness on SiO 2 / Si wafer using optical-fiber-type low-coherence interferometry

J. Appl. Phys. 105, 013110 (2009); 10.1063/1.3058592

Improving the reliability of multiplexed fiber optic low-coherence interferometric sensors by use of novel twinloop network topologies

Rev. Sci. Instrum. 78, 055106 (2007); 10.1063/1.2735564

Photorefractive coherence-gated interferometry

Rev. Sci. Instrum. 69, 3741 (1998); 10.1063/1.1149172

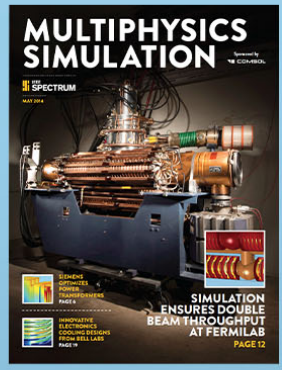




\title{
Resolving interparticle position and optical forces along the axial direction using optical coherence gating
}

\author{
T. H. Chow, ${ }^{1,2, a)}$ W. M. Lee, ${ }^{3, a), b)}$ K. M. Tan, ${ }^{4}$ B. K. Ng, ${ }^{1, a)}$ and C. J. R. Sheppard ${ }^{5}$ \\ ${ }^{1}$ School of Electrical and Electronic Engineering, Nanyang Technological University, 50 Nanyang Ave., \\ Block S1, Singapore 639798 \\ ${ }^{2}$ Singapore-MIT Alliance, Center for Singapore-MIT Alliance, National University of Singapore, E4-04-10, \\ 4 Engineering Drive 3, Singapore 117576 \\ ${ }^{3}$ Einst Technology Pte Ltd., 1092 Lower Delta Road \#04-01, Tiong Bahru Industrial Estate, \\ Singapore 169203 \\ ${ }^{4}$ SUPA, School of Physics and Astronomy, University of St. Andrews, North Haugh, St. Andrews KY16 9SS, \\ United Kingdom \\ ${ }^{5}$ Division of Bioengineering, National University of Singapore, 7 Engineering Drive 1, Singapore 117574
}

(Received 9 June 2010; accepted 12 October 2010; published online 10 December 2010)

\begin{abstract}
In this paper, we demonstrate the use of coherence gating to resolve particle positions and forces in the axial direction. Through coherence gating, particle displacements and interparticle separations can be resolved with a high signal-to-noise ratio. We achieved both high depth resolvability $\left(10^{-6} \mathrm{~m}\right)$ and weak optical force $\left(10^{-15} \mathrm{~N}\right)$ measurements in an optical trapping system using a low

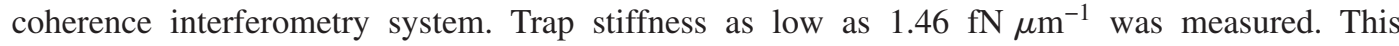
technique is well-suited for the direct visualization of interparticle optical-mechanical interactions. (C) 2010 American Institute of Physics. [doi:10.1063/1.3519976]
\end{abstract}

Back focal plane interferometry (BFP) system can provide position sensing down to the subnanometer range and is the most commonly used high resolution interferometric tracking method in optical trapping. ${ }^{1-3}$ The high sensitivity of the BFP technique comes with the tradeoff of a limited axial tracking range of about a few micrometers, ${ }^{3}$ and more importantly, the inability to discern two or more particles residing along the axial direction of a single optical trap. ${ }^{4}$ This is necessary for the detection and quantification of multiple particle dynamics in applications such as optical binding, ${ }^{5}$ optical assembly structures $^{6}$ and optical chromatography. ${ }^{7}$

Grier and co-workers ${ }^{8}$ developed an alternative approach for monitoring multiple optically trapped particles over tens of micrometers using the interference pattern from the farfield scattering of the trapped particles. ${ }^{8}$ Although this approach overcomes the limitations of the BFP, one of its caveats is the need for an accurate model to describe the interference of the scattered light fields and effects such as light refocusing between the trapped particles. Furthermore, the interference of highly coherent light sources is also susceptible to subtle optical distortion introduced along the interference path. Hence, there will be some level of uncertainty in obtaining a direct measure of the optical centers of multiple trapped particles using the holographic particle tracking technique.

In optical binding, measuring weak, subpiconewton interaction forces between multiple axially trapped particles have revealed intricate nonlinear dynamics ${ }^{9}$ due to the rescattering of light among the particles. Understanding the dy-

\footnotetext{
a) Authors to whom correspondence should be addressed. Electronic addresses: chow0035@e.ntu.edu.sg, Lee.WoeiMing@mgh.harvard.edu, and ebkng@ntu.edu.sg.

${ }^{b}$ Present address: Harvard Medical School \& Massachusetts General Hospital, Wellman Center for Photomedicine, 40 Blossom St., Boston MA 02114 .
}

namics between multiple trapped particles is also important for the development of complex light-induced self assembled structures. ${ }^{10}$ Reflectance mode low coherence interferometry (LCI) for microscopy imaging have provided high resolution tomography images of cells ${ }^{11}$ and is capable of measuring very subtle changes in the refractive indices. ${ }^{12}$ Through coherence gating, particle displacements can be resolved with high signal-to-noise ratio due to the rejection of photons whose optical path differences are beyond the coherence length of the light source. With this approach, displacements down to the picometers can be measured. ${ }^{13}$

In this paper, we demonstrate the unique advantage of using coherence gating to determine the positions and trapping forces of optically trapped particles. A common-path system is successfully integrated with a single beam optical trapping system to determine interparticle separation with accuracy in the order of the light source's coherence length. The optical centers of two particles that are axially aligned in a single optical trap can clearly be discerned in the integrated LCI-trapping system. The optical trapping forces are inferred from the time-varying displacement of the particle's optical center from its mean equilibrium.

Figure 1 shows the experiment setup of an integrated LCI-trapping system sharing the same optical path through a 0.4 NA microscope objective (NA denotes numerical aperture). Due to the weak optical trap, only the $10 \mu \mathrm{m}$ microspheres can be levitated while the $50 \mu \mathrm{m}$ microspheres remains at the base of the cavity to serve as the secondary stationary spheres, and refocusing microlenses in the experiments. The lateral positions of the microspheres were monitored with the aid of a charged coupled device (CCD) camera.

In our experiment, the scattering forces from the trapping beam guide the particles in the longitudinal direction and the corresponding gradient forces draw the particle into the beam. ${ }^{3}$ A weak equilibrium position ${ }^{14}$ of the microsphere is obtained when the upward forces due to scattering forces 


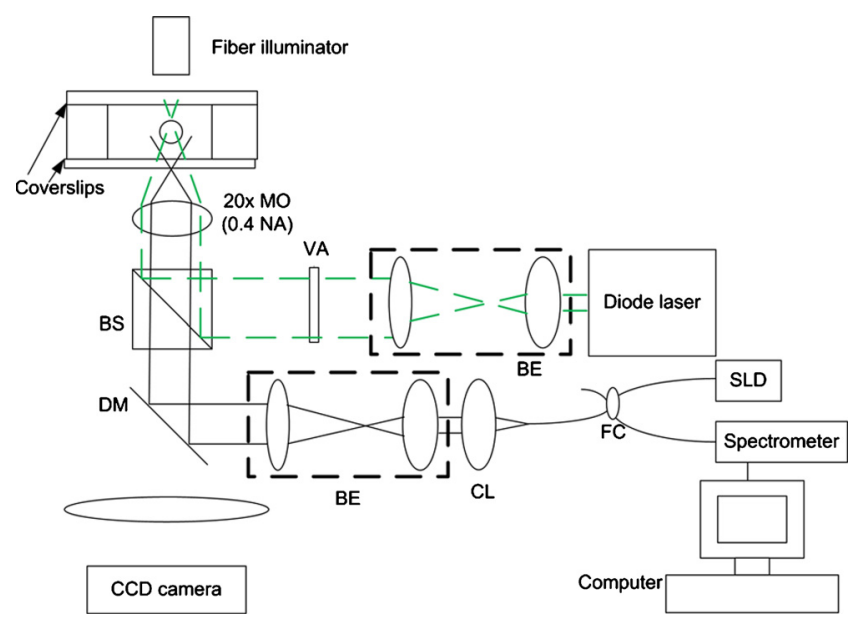

FIG. 1. (Color online) Schematic diagram of collinear low coherence interferometry sensing system consisting of the following components: MO: microscope objective, BS: beamsplitter, VA: variable attenuator, DM: dichroic mirror, BE: beam expansion optics, CL: collimating lens, FC: fiber coupler $(2 \times 2)$, SLD: superluminescence diode $\left(\lambda_{\text {center }}=820 \mathrm{~nm}, \Delta \lambda=100 \mathrm{~nm}\right.$, and theoretical axial resolution of $2.8 \mu \mathrm{m}), \mathrm{CCD}$ camera, fiber illuminator, and diode laser. $\left(\lambda=532 \mathrm{~nm}\right.$ and $\left.\mathrm{P}_{\max }=50 \mathrm{~mW}\right)$. Laser power fluctuation is less than $1 \%$ during measurements. A weak optical trap is formed using a 0.4 NA objective lens (Edmund Optics), Edmund Optics Singapore Pte. Ltd., 1093 Lower Delta Road \#05-10 Tiong Bahru Industrial Estate Singapore 169204. The trapping beam is expanded (telecentric) to ensure that the back aperture of the objective lens is overfilled. A spectrometer (Ocean Optics, HR4000), 830 Doublas Ave., Dundein, FL 34698, USA with 3648 pixels covering a wavelength range of 759-961 nm gave an imaging depth of $3.3 \mathrm{~mm}$ in air. A custom-made cavity of $1 \mathrm{~mm}$ in depth is filled with water and polystyrene microspheres (PolySciences, Inc.), 400 Valley Road, Warrington, PA 18976 of 10 and $50 \mu \mathrm{m}$ diameters.

and buoyancy balances the weight of the microsphere. ${ }^{15}$ At its equilibrium position, the trapped particle can be considered a harmonic oscillator in an overdamped system with an associated stiffness. ${ }^{3}$ The axial trap stiffness can be increased by increasing the numerical aperture of the trapping optics. The value of the trap stiffness is sensitive to changes in the geometrical intensity distribution in the optical trap.

As both the reference and sample signals share the same optical path, dispersion and polarization mismatches between them are minimized. ${ }^{16}$ The sample signal, arising from reflecting surfaces of microspheres in the optical trap, interferes with the reference signal from the first cover slip to give a modulated spectrum of the superluminescence diode (SLD). The positions of the two reflecting surfaces of the microsphere are determined by taking the inverse Fourier transform of the detected spectrum. In our measurements, the Rayleigh length of the LCI beam was optimized for the particle diameter to accurately determine the reflecting surfaces of the microsphere. ${ }^{17}$ The measured spectrum is zero-padded to 16384 points to minimize discretization errors and a positional sensitivity of $\pm 217 \mathrm{~nm}$ is achieved.

Figure 2 shows the LCI imaging results of a levitating $10 \mu \mathrm{m}$ sphere as a function of time. The power of the diode laser after the microscope objective was found to be $10 \mathrm{~mW}$. As shown in Fig. 2(a), the surfaces of the $10 \mu \mathrm{m}$ microsphere $\left(n_{\text {microsphere }}=1.59\right)$ along the axial direction is represented by two successive bright lines separated by an optical path difference of about $16 \mu \mathrm{m}$ as the microsphere rises in the cavity. The optical center (OC) of the trapped particle is determined from the mean physical positions of the microsphere surfaces. While the microsphere shows effects of defocusing in the brightfield images (Fig 2(a)) as it rises in the
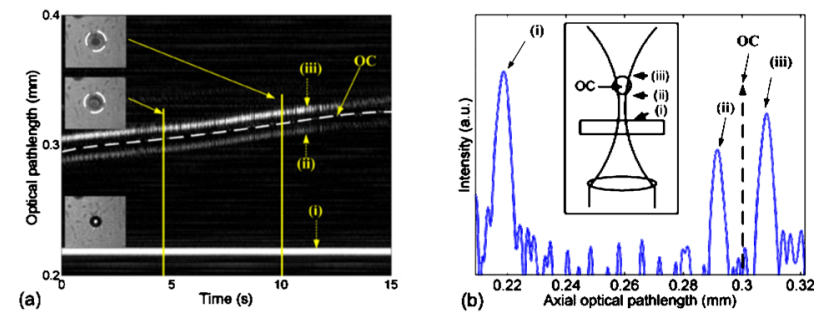

FIG. 2. (Color online) (a) Low coherence image of a levitating $10 \mu \mathrm{m}$ microsphere (the dashed line shows the position of the optical center OC) The insets show CCD images of the microspheres at different axial positions, defocusing effects are clearly seen, (b) depth resolved intensity profile at time point corresponding to the middle CCD image, inset shows the optical setup. The dashed arrows marked the position of the coverslip (i) and reflecting surface of the microspheres (ii) and (iii).

cavity the microsphere surfaces can still be observed at these time points in the LCI image. This illustrates the long range tracking capability of LCI.

Figure 3(a) shows the surfaces of multiple microspheres imaged by LCI. The depth-resolved intensity profile of an optically trapped, $10 \mu \mathrm{m}$ microsphere positioned above a $50 \mu \mathrm{m}$ microsphere situated on a cover slip demonstrates the capability of the system to image more than one particle in an optical trap [refer to Fig. 3(b)]. The physical distance between the particles is measured to be about $19 \mu \mathrm{m}$ at an optical trapping power of about $2.3 \mathrm{~mW}$. The refocusing of light after passing through the $50 \mu \mathrm{m}$ microsphere resulted in a smaller trapping power than the maximum available power.

The microsphere dynamics is determined by monitoring its optical center with LCI. The dynamics of a microsphere without being optically trapped is Brownian motion in nature and can be characterized by observing its mean-square position as described by $\left\langle x^{2}\right\rangle=2 D t$, where the diffusion constant $D=k_{B} T / 6 . \pi . n . r, n$ is the viscosity, $r$ is the microsphere radius, and $t$ is the duration of observation $\left(k_{B} T\right.$ $=4.1 \mathrm{fN} \mu \mathrm{m}, \mathrm{T}=300 \mathrm{~K})$. This gives a free diffusion constant and a mean-square displacement of $0.826 \mu \mathrm{m}^{2} \mathrm{~s}^{-1}$ and $1.65 \mu \mathrm{m}^{2}$, respectively, for a $10 \mu \mathrm{m}$ microsphere over a period of $1 \mathrm{~s}$. Our LCI is capable of measuring the dynamics motion of the $10 \mu \mathrm{m}$ microsphere since the position displacement is within the linear detection range of our system (position sensitivity of $0.217 \mu \mathrm{m}$ and linearity of over $100 \mu \mathrm{m})$. In the presence of a weak optical trap, the stiffness, $k_{\text {trap }}$ of a trapped $10 \mu \mathrm{m}$ microsphere can be computed from equipartition theorem where $k_{\text {trap }}=k_{B} T /\left\langle x^{2}\right\rangle$. To determine $\left\langle x^{2}\right\rangle$ with respect to the equilibrium position, the optical center of the $10 \mu \mathrm{m}$ microsphere is monitored using the LCI

(a)

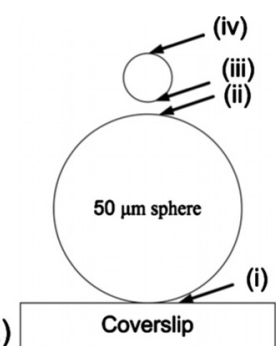

(b)

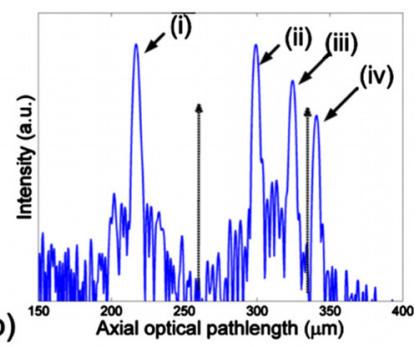

FIG. 3. (Color online) (a) Schematic diagram of the 10 and $50 \mu \mathrm{m}$ microspheres in a weak optical trap. (b) Depth-resolved intensity profile of the two trapped microspheres. The dotted arrows marked the optical centers of the micrespheres, (i), (ii), (iii), and (iv) indicates the reflecting surfaces 


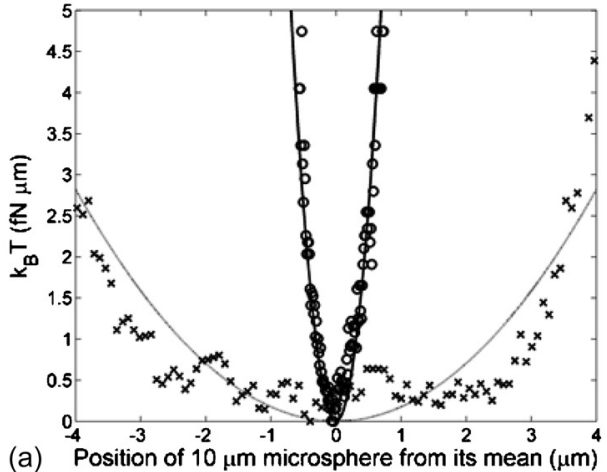

(a) Position of $10 \mu \mathrm{m}$ microsphere from its mean $(\mu \mathrm{m})$

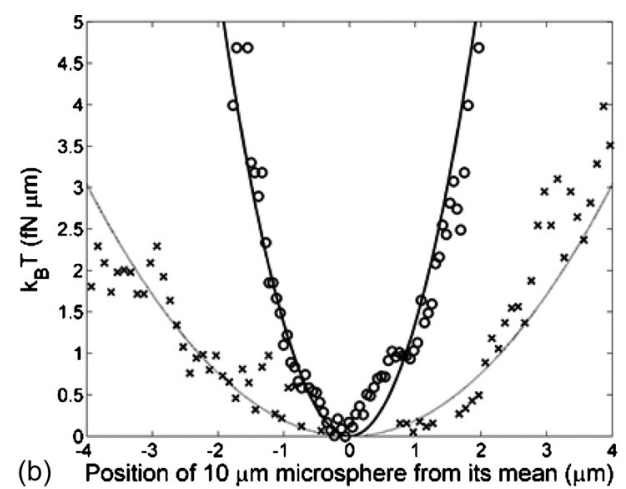

FIG. 4. Optical potential profiles of a $10 \mu \mathrm{m}$ microsphere measured with (a) objective lenses of $0.4\left(\times, k_{0.4 \mathrm{NA}}\right.$

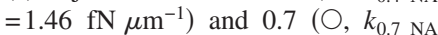

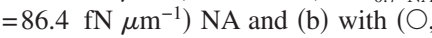

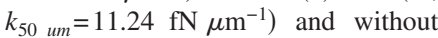
$\left(\times, \quad k_{\text {no } 50 u m}=1.57 \mathrm{fN} \mu \mathrm{m}^{-1}\right), \quad$ a $50 \mu \mathrm{m}$ microsphere in the optical trap. The corresponding fitted optical potential profiles (lines) are also shown in the figure. The fitting accuracy as quantified by $\mathrm{R}$-square is at least 0.82 in the experiments. system over a period of $10 \mathrm{~min}$. With a sampling time of 100 $\mathrm{ms}, 6,000$ data points were obtained. The probability of the microsphere's displacement in a potential well, modeled as a Boltzmann distribution, ${ }^{3}$ is obtained from the histogram of these data points. The trapping potential energy, $U(x)$, is determined by taking the logarithm of the probability. The potential energy, having a quadratic dependence on the displacement, is then fitted to a quadratic function to determine the trap stiffness. ${ }^{3}$ Figure 4(a) shows the optical potential energy of a trapped $10 \mu \mathrm{m}$ microsphere obtained from the LCI system using two different NA objectives but with the same optical power of $2.3 \mathrm{~mW}$. When the NA of the objective is increased from 0.4 to 0.7 , the trap stiffness is in-

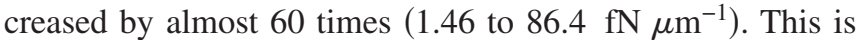
in agreement with the higher axial intensity gradient expected in the optical trap from the use of a larger NA objective.

Besides changing the NA of the objective, the effect of microsphere lensing on the axial trap stiffness was also investigated. The refocusing of an initial trapping beam through a microsphere can strongly influence the dynamics of the trapped particle through longitudinal optical binding. ${ }^{9}$ In our experiment, a single stationary $50 \mu \mathrm{m}$ microsphere, illustrated in Fig. 3(a), is used as the refocusing element. The $50 \mu \mathrm{m}$ microsphere acts as a ball lens that refocuses the trapping beam to yield a shorter focal length and increases the NA of the trapping system. The trapping position of the $10 \mu \mathrm{m}$ microsphere changed from 65 to $170 \mu \mathrm{m}$ above the cavity floor when the $50 \mu \mathrm{m}$ microsphere is removed. Figure 4(b) shows the optical potential profiles of the $10 \mu \mathrm{m}$ microsphere with and without the $50 \mu \mathrm{m}$ microsphere. The measured trap stiffness is observed to increase by 7 times

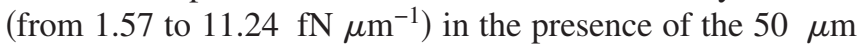
microsphere, as expected from the resulting increase in the effective NA of the optical trap. The measurements of both the absolute positional shift of the $10 \mu \mathrm{m}$ microsphere and the increased trap stiffness due to the refocusing of light by the $50 \mu \mathrm{m}$ microsphere highlights the strength of LCI in the direct visualization of interparticle optical-mechanical interactions. ${ }^{9}$ The results in Fig. 4(b) elucidate the use of LCI in the measurement of interparticle dynamics.
In conclusion, we have demonstrated the capability of coherence gating in a common-path LCI-trapping system for measuring positional fluctuations of microspheres. The functionality of LCI in optical trapping is especially useful for studying multiparticle interactions in optical binding, ${ }^{9}$ in situ measurement of different particle separation in optical chromatography $^{7}$ and cell deformation in an optical cell stretcher. ${ }^{8}$ The common-path approach can easily be incorporated into any optical trapping platform, especially in optical fiber trapping systems ${ }^{9}$ with minimized dispersion and polarization mismatches.

The authors acknowledge initial discussions with Professor Kishan Dholakia. This research is supported by grants (SCS-BU0052 and RP C-015/2007) from Agency for Science, Technology and Research and grant (RGM39/06) from the Ministry of Education, Singapore.

${ }^{1}$ F. Gittes and C. F. Schmidt, Opt. Lett. 23, 7 (1998).

${ }^{2}$ S. Perrone, G. Volpe, and D. Petrov, Rev. Sci. Instrum. 79, 106101 (2008).

${ }^{3}$ K. C. Neuman and S. M. Block, Rev. Sci. Instrum. 75, 2787 (2004).

${ }^{4} \mathrm{M}$. Li and J. Arlt, Opt. Commun. 281, 135 (2008).

${ }^{5}$ M. M. Burns, J. M. Fournier, and J. A. Golovchenko, Phys. Rev. Lett. 63, 1233 (1989).

${ }^{6}$ M. P. MacDonald, L. Paterson, K. Volke-Sepulveda, J. Arlt, W. Sibbett, and K. Dholakia, Science 296, 1101 (2002).

${ }^{7}$ S. J. Hart and A. V. Terray, Appl. Phys. Lett. 83, 5316 (2003).

${ }^{8}$ F. C. Cheong, B. J. Krishnatreya, and D. G. Grier, Opt. Express 18(13), 13563 (2010).

${ }^{9}$ N. K. Metzger, R. F. Marchington, M. Mazilu, R. L. Smith, K. Dholakia, and E. M. Wright, Phys. Rev. Lett. 98, 068102 (2007).

${ }^{10}$ K. Dholakia and P. Zemanek, Rev. Mod. Phys. 82(2), 1767 (2010).

${ }^{11}$ W. Tan, A. L. Oldenburg, J. J. Norman, T. A. Desai, and S. A. Boppart, Opt. Express 14, 7159 (2006).

${ }^{12}$ C. Yang, A. Wax, M. S. Hahn, K. Badizadegan, R. R. Dasari, and M. S. Feld, Opt. Lett. 26, 1271 (2001).

${ }^{13}$ C. Joo, T. Akkin, B. Cense, B. H. Park, and J. F. De Boer, Opt. Lett. 30, 2131 (2005).

${ }^{14}$ The weight of a $10 \mu \mathrm{m}$ polystyrene sphere is about $5.4 \mathrm{pN}$. Accounting for buoyancy forces, the net downward force is $250 \mathrm{fN}$. Thus the upward force due to the optical scattering is also $250 \mathrm{fN}$ (Refs. 7 and 18).

${ }^{15}$ A. Ashkin, Phys. Rev. Lett. 24, 156 (1970).

${ }^{16}$ A. B. Vakhtin, D. J. Kane, W. R. Wood, and K. A. Peterson, Appl. Opt. 42, 6953 (2003).

${ }^{17}$ A. Ashkin and J. M. Dziedzic, Appl. Opt. 20, 1803 (1981).

${ }^{18}$ J. Guck, R. Ananthakrishnan, C. C. Cunningham, and J. Käs, J. Phys.: Condens. Matter 14, 4843 (2002). 